\title{
Influence of Magnetic Field Direction on Rearrangement of Martensite Variants in an Fe-Pd Alloy
}

\author{
Tatsuaki Sakamoto $^{1, *}$, Takashi Fukuda ${ }^{1}$, Tomoyuki Kakeshita ${ }^{1}$, Tetsuya Takeuchi ${ }^{2}$ and Kohji Kishio $^{3}$ \\ ${ }^{1}$ Department of Materials Science and Engineering, Graduate School of Engineering, Osaka University, Suita 565-0871, Japan \\ ${ }^{2}$ Low Temperature Center, Osaka University, Toyonaka 560-0043, Japan \\ ${ }^{3}$ Department of Superconductivity, Graduate School of Engineering, University of Tokyo, Tokyo 113-8656, Japan
}

Influence of magnetic field direction on rearrangement of martensite variants in an Fe-31.2Pd(at\%) single crystal has been investigated using thermal expansion measurement in a cooling process under the magnetic field applied along $[001]_{\mathrm{P}},[011]_{\mathrm{P}}$ and $[111]_{\mathrm{P}}($ " $\mathrm{P}$ " represents "parent" phase). We also observe these rearrangements of variants in the cooling processes under the magnetic field applied along $[001]_{\mathrm{P}},[011]_{\mathrm{P}}$ and $[111]_{\mathrm{P}}$ by optical microscope. From the thermal expansion measurement and the optical microscope observation, the following results are obtained: the variants are rearranged almost perfectly into the variants whose $a$ axes are parallel to the magnetic field applied along [001 $]_{\mathrm{P}}$, imperfectly into the variants whose $c$ axis is perpendicular to the magnetic field applied along [011 $]_{\mathrm{P}}$ and hardly along [111 $]_{\mathrm{P}}$. We explain these results considering the following criterion: rearrangement of variants proceeds when a kind of shear stress generated by applying a magnetic field acting on the twinning plane is larger than the stress required for the rearrangement of variants.

(Received August 7, 2003; Accepted September 26, 2003)

Keywords: iron-palladium alloy, ferromagnetic shape memory alloy, magnetic field-induced strain, rearrangement of variants, magnetocrystalline anisotropy

\section{Introduction}

Ferromagnetic shape memory alloys have attracted a considerable attention because these alloys exhibit a large strain by applying a magnetic field, and its value is larger than that of conventional magnetostrictive materials. ${ }^{1-3)}$ However the mechanism of the magnetic field-induced strain (MFIS) in the ferromagnetic shape memory alloys is substantially different from that of conventional magnetostrictive materials. That is, the former is caused by rearrangement of variants, but the latter is not. Thus the mechanism of rearrangement of variants by applying magnetic field is of much interest, and a lot of studies have been made so far. ${ }^{4-10)}$

According to Ullakko et al., rearrangement of variants occurs when magnetic anisotropy energy is larger than the energy required for the rearrangement of variants. ${ }^{4)}$ However the propriety of this criterion has not been well confirmed yet. Thus it is necessary to examine influence of magnetic field direction on rearrangement of variants in a single crystal because a criterion for rearrangement of variants should be satisfied regardless of the direction of a magnetic field. However in most studies reported so far, rearrangement of variants has been examined along $[001]_{\mathrm{P}}$ of a Ni-Mn-Ga alloy ${ }^{4,6,8,9)}$ and there is no report in which influence of magnetic field direction on rearrangement of variants is examined systematically. In the present paper we investigate rearrangement of variants by applying a magnetic field along main crystallographic orientations in a single crystal, i.e., $[001]_{\mathrm{P}},[011]_{\mathrm{P}}$ and $[111]_{\mathrm{P}}$, and discuss the mechanism of rearrangement of variants by applying a magnetic field.

For this research, we selected a disordered Fe-31.2Pd(at\%) alloy, partly because in this alloy we confirmed that rearrangement of variants occurs under the magnetic field ${ }^{11)}$ after the first discovery in Fe-30Pd by James and Wuttig, ${ }^{5)}$

*Graduate Student, Osaka University and partly because we know detailed crystallographic features of this alloy as follows: A disordered Fe-Pd alloy containing about 30 at\% Pd undergoes a thermoelastic transformation from a f.c.c. parent phase to a tetragonal, so-called f.c.t., martensite phase. ${ }^{12)}$ In the martensite phase there exist three martensite variants whose $c$ axes are parallel to $[001]_{\mathrm{P}},[010]_{\mathrm{P}}$ and $[100]_{\mathrm{P}}$, respectively, and the $a$ axes (easy axes of magnetization) of three variants are also parallel to $\langle 001\rangle_{\mathrm{P}}$. Two neighboring variants are in twin relationship, and have a twinning plane of $\{011\}_{\mathrm{M}}{ }^{12)}$ ("M" represents "martensite" phase).

\section{Experimental Procedure}

An ingot of $\mathrm{Fe}-31.2 \mathrm{Pd}$ was prepared from high purity iron (99.99\%) and palladium (99.97\%) by arc-melting in argon atmosphere. This ingot was grown into a single crystal by floating zone method in argon atmosphere. Two rectangular parallelepiped specimens were cut from the single crystal. One has the dimension of $3.7 \mathrm{~mm}$ along $[001]_{\mathrm{P}}, 3.1 \mathrm{~mm}$ $[011]_{\mathrm{P}}$ and $3.1 \mathrm{~mm}[01 \overline{1}]_{\mathrm{P}}$ (specimen $\left.\mathrm{A}\right)$. The other has the dimension of $1.6 \mathrm{~mm}$ along $[111]_{\mathrm{P}}, 1.9 \mathrm{~mm}[1 \overline{1} 0]_{\mathrm{P}}$ and $2.1 \mathrm{~mm}[11 \overline{2}]_{\mathrm{P}}$ (specimen B). These specimens were homogenized at $1373 \mathrm{~K}$ for $24 \mathrm{~h}$, followed by quenching into iced water. Thermal expansion was measured under the magnetic field along $[001]_{\mathrm{P}},[011]_{\mathrm{P}}$ by using specimen A and along $[111]_{P}$ by using specimen B. Figure 1 shows the schematic illustration of two specimens for the thermal expansion measurement under the magnetic field. In the thermal expansion measurement under the magnetic field applied along $[001]_{\mathrm{P}}$ and $[011]_{\mathrm{P}}$, the strain was measured along the field direction by three terminal capacitance method. In the thermal expansion measurement under the magnetic field applied along $[111]_{\mathrm{P}}$, the strain was measured along $[001]_{\mathrm{P}}$ on $(1 \overline{10})_{\mathrm{P}}$ instead of the field direction because the strain along $[111]_{\mathrm{P}}$ is not detectable regardless of the rearrangement 


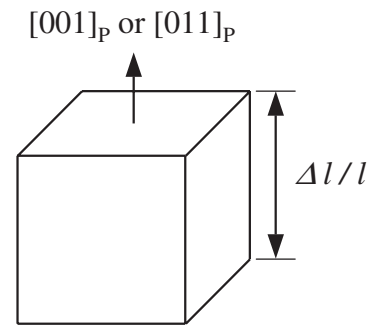

(a) specimen $\mathrm{A}$

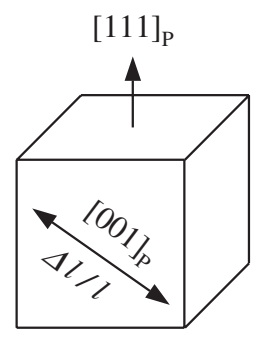
$H / /[111]_{\mathrm{P}}$ (b) specimen B

Fig. 1 Illustration of specimens for thermal expansion measurement. The directions of the applied magnetic field and the strain measurement are shown.

of variants. To measure the strain of specimen $B$, we used a strain gauge method instead of the capacitance method because in the capacitance method, a strain is measured along the direction parallel to an edge of a specimen but no edge of specimen $\mathrm{B}$ is parallel to $[001]_{\mathrm{P}}$. Optical microscope observation under the magnetic field along $[001]_{\mathrm{P}},[011]_{\mathrm{P}}$ or $[111]_{P}$ was carried out by using a Nomalski-type differential interference contrast method. In the thermal expansion measurement and the optical microscope observation under the magnetic field, the bottom of the specimen was fixed to the stage by using a vinyl phenolic adhesive so as to prevent rotational movement of the specimen under the magnetic field.

\section{Results}

\subsection{Magnetic field-induced strain measurement}

To examine influence of magnetic field direction on rearrangement of variants, thermal expansions were investigated during the cooling process under the magnetic field applied along $[001]_{\mathrm{P}},[011]_{\mathrm{P}}$ and $[111]_{\mathrm{P}}$, respectively. The specimens were cooled down from room temperature to $77 \mathrm{~K}$ under the magnetic field of $3.2 \mathrm{MA} / \mathrm{m}$, which is sufficient to saturate the magnetization, and the results are shown in Fig. 2.

On cooling under the magnetic field applied along $[001]_{\mathrm{P}}$, the strain along $[001]_{\mathrm{P}}$ begins to increase at about $T_{\mathrm{M}}=230 \mathrm{~K}$ and reaches $2.0 \%$ at $77 \mathrm{~K}$. By using the value of this strain, we calculate the total fraction, $f_{a \| H}$, of the two variants with the $a$ axes parallel to the magnetic field along $[001]_{\mathrm{P}}$. This fraction $f_{a \| H}$ is calculated because $f_{a \| H}$ increases with increasing the strain. The fraction $f_{a \| H}$ is obtained through the following equation,

$$
f_{a \| H} a_{T}+\left(1-f_{a \| H}\right) c_{T}=a_{300 \mathrm{~K}}\left(1+(\Delta l / l)_{\mathrm{cool}}\right),
$$

where $a_{T}$ and $c_{T}$ are the lattice parameters of the martensite at temperature $T, a_{300 \mathrm{~K}}$ is that of the parent phase at $300 \mathrm{~K}$ and $(\Delta l / l)_{\mathrm{cool}}$ is a strain due to the thermal expansion obtained when the specimen is cooled down from 300 to $T \mathrm{~K}$. The fraction $f_{a \| H}$ calculated by using the lattice parameters obtained in a previous paper ${ }^{11)}$ is shown in Fig. 3. As a result, $f_{a \| H}$ is almost $100 \%$ at any temperature where the measurement was carried out. Incidentally, from the viewpoint of magnetocrystalline anisotropy, this selected variant is the one whose uniaxial magnetocrystalline anisotropy energy is the

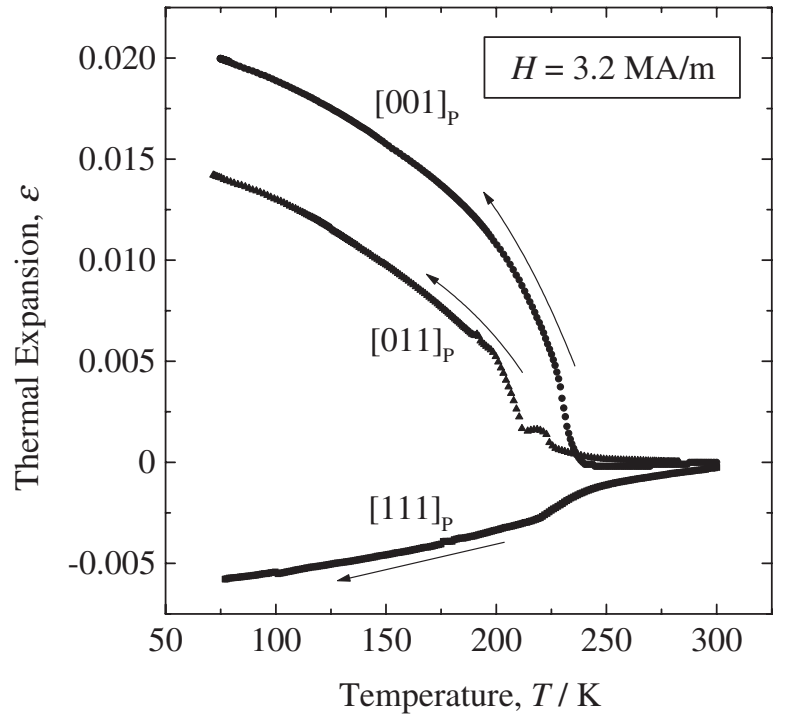

Fig. 2 Thermal expansion under the magnetic field of $3.2 \mathrm{MA} / \mathrm{m}$ applied along $[001]_{\mathrm{P}},[011]_{\mathrm{P}}$ and $[111]_{\mathrm{P}}$ of $\mathrm{Fe}-31.2 \mathrm{Pd}(\mathrm{at} \%)$.

lowest under the magnetic field applied along $[001]_{\mathrm{P}}$ because the $a$ axis is the easy axis.

When a magnetic field is applied along $[011]_{\mathrm{P}}$, the strain also begins to increase at about $T_{\mathrm{M}}$ as shown in Fig. 2. The strain reaches only $1.4 \%$ at $77 \mathrm{~K}$. The fraction, $f_{c \perp H}$, of the variant with $c$ axis perpendicular to the magnetic field along $[011]_{\mathrm{P}}$ can be calculated by

$$
f_{c \perp H} a_{T}+\left(1-f_{c \perp H}\right)\left(a_{T}+c_{T}\right) / 2=a_{300 \mathrm{~K}}\left(1+(\Delta l / l)_{\mathrm{cool}}\right) .
$$

We calculated $f_{c \perp H}$ because $f_{c \perp H}$ increases with increasing the strain. The fraction $\left(1-f_{c \perp H}\right)$ is the sum of the fraction of the two variants whose $c$ axes form an angle of $\pi / 4$ with the magnetic field. The calculated fraction is shown in Fig. 3. According to this calculation, $f_{c \perp H}$ is $49 \%$ at $210 \mathrm{~K}$, and gradually increases to $86 \%$ as temperature decreases to $77 \mathrm{~K}$. However $f_{c \perp H}$ does not reach $100 \%$. Incidentally, this selected variant is also the one whose uniaxial magnetocrystalline anisotropy energy is the lowest under the magnetic field applied along $[011]_{\mathrm{P}}$.

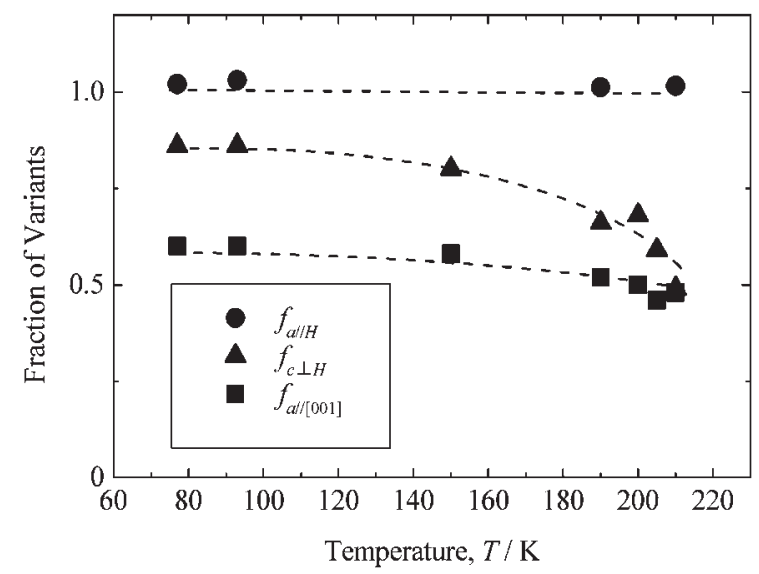

Fig. 3 The calculated fraction of variants, $f_{a \| H}, f_{c \perp H}$ and $f_{a \|[001]}$, under the magnetic field of $3.2 \mathrm{MA} / \mathrm{m}$ applied along $[001]_{\mathrm{P}},[011]_{\mathrm{P}}$ and $[111]_{\mathrm{P}}$, respectively, of $\mathrm{Fe}-31.2 \mathrm{Pd}(\mathrm{at} \%)$. Broken lines are guides for eyes. 

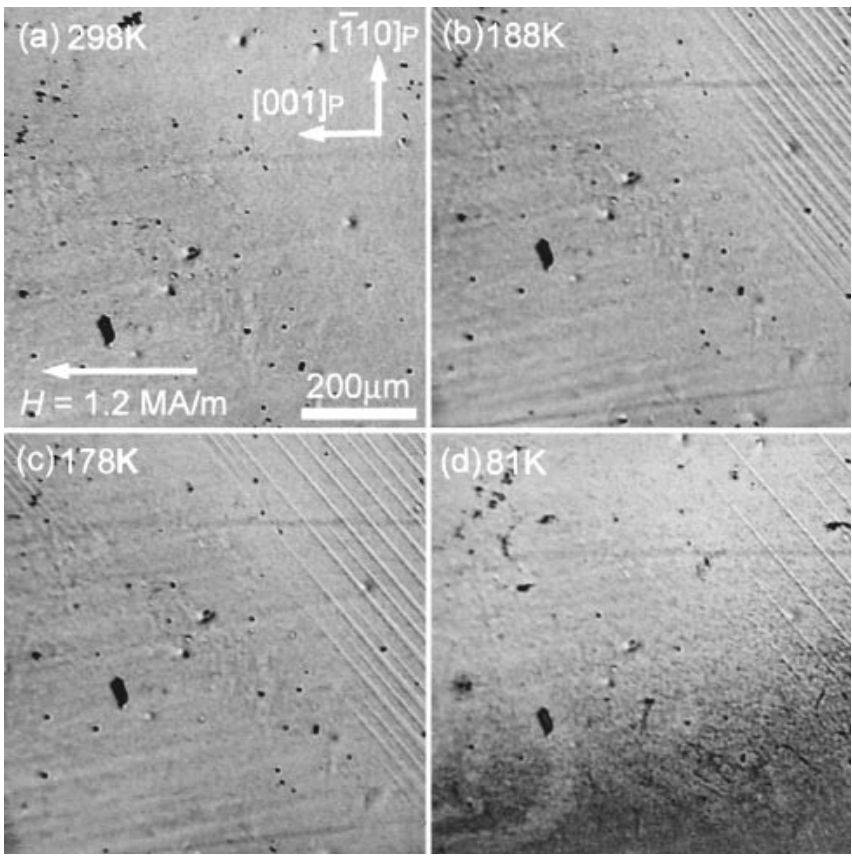

Fig. 4 A series of optical micrographs of $\mathrm{Fe}-31.2 \mathrm{Pd}(\mathrm{at} \%)$ in the cooling process under the magnetic field of $1.2 \mathrm{MA} / \mathrm{m}$ along the $[001]_{\mathrm{P}}$ direction: the parent phase is shown in (a); the martensite phase is shown in (b), (c) and (d).

On cooling under the magnetic field applied along $[111]_{\mathrm{P}}$, the specimen begins to contract along $[001]_{\mathrm{P}}$ at about $T_{\mathrm{M}}$ and the strain reaches $-0.58 \%$ at $77 \mathrm{~K}$. The total fraction, $f_{a \|[001]}$, of the two variants with $a$ axes along $[001]_{\mathrm{P}}$ can be calculated by

$$
f_{a \|[001]} a_{T}+\left(1-f_{a \|[001]}\right) c_{T}=a_{300 \mathrm{~K}}\left(1+(\Delta l / l)_{\mathrm{cool}}\right) .
$$

We calculated $f_{a \|[001]}$ because the strain was measured along $[001]_{\mathrm{P}}$. As a result, $f_{a \|[001]}$ is $60 \%$. This means that when a magnetic field is applied along $[111]_{\mathrm{P}}$, rearrangement of variants hardly occurs because the calculated fraction is nearly equal to two thirds, which is expected naturally if three variants are equivalently introduced. The small deviation from the expected value may arise because defects in the specimen influence the rearrangement of variants. This result is consistent with the fact speculated from the point of view of magnetocrystalline anisotropy that no specific variant is selected because the difference of the magnetocrystalline anisotropy energy among variants is zero under the magnetic field applied along $[111]_{\mathrm{P}}$.

\subsection{Optical microscope observation}

Rearrangement of variants was directly observed by optical microscope. A series of micrographs taken in the cooling process under the magnetic field of $1.2 \mathrm{MA} / \mathrm{m}$ (this strength of the magnetic field is sufficient to saturate the magnetization) parallel to $[001]_{\mathrm{P}}$ is shown in Fig. 4. Above $T_{\mathrm{M}}$, the surface of the specimen is flat in the parent phase [Fig. 4(a)]. After the temperature decreases below $T_{\mathrm{M}}$, a striped contrast of surface relief partially appears [Fig. 4(b)]. On further cooling, one band of the surface relief becomes narrower and the other wider [Fig. 4(c)]. Finally, the surface relief disappears almost perfectly [Fig. 4(d)]. This result is consistent with that of the thermal expansion measurement
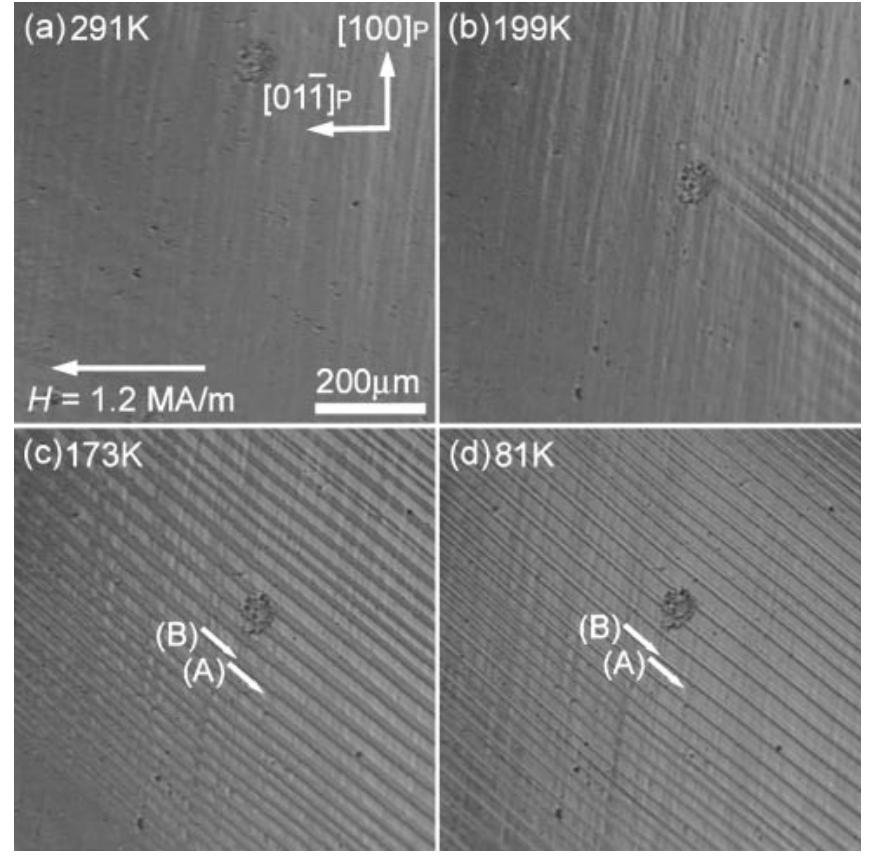

Fig. 5 A series of optical micrographs of Fe-31.2Pd(at\%) in the cooling process under the magnetic field of $1.2 \mathrm{MA} / \mathrm{m}$ along the $[011]_{\mathrm{P}}$ direction: the parent phase is shown in (a); the martensite phase is shown in (b), (c) and (d).

under the magnetic field applied along $[001]_{P}$ as shown in Fig. 3.

The observation under magnetic field of $1.2 \mathrm{MA} / \mathrm{m}$ along $[011]_{\mathrm{P}}$ is shown in Fig. 5. In the parent phase, the surface of the specimen is flat [Fig. 5(a)]. Below $T_{\mathrm{M}}$, the surface relief appears [Fig. 5(b)]. On further cooling, the region of striped contrast spreads [Fig. 5(c)], and the ratio of the band width of region (A) to that of region (B) gradually changes from nearly $5: 5$ at $173 \mathrm{~K}$ to nearly $7: 3$ at $81 \mathrm{~K}$. However the contrast of the surface relief does not disappear perfectly [Fig. 5(d)]. This result is also consistent with that of the thermal expansion measurement under the magnetic field applied along [011 $]_{\mathrm{P}}$ as shown in Fig. 3.

Figure 6 shows the result of observation under the magnetic field of $1.2 \mathrm{MA} / \mathrm{m}$ along [111 $]_{\mathrm{P}}$. The surface of the specimen is flat above $T_{\mathrm{M}}$ [Fig. 6(a)], and the surface relief appears below $T_{\mathrm{M}}$ [Fig. 6(b)]. As the specimen is cooled down, the contrast of the surface relief becomes sharper and sharper [Figs. 6(c), (d)]. However, the configuration of the variants does not change. Accordingly, the variants are hardly rearranged when a magnetic field is applied along $[111]_{\mathrm{P}}$. This result is also consistent with that of the thermal expansion measurement under the magnetic field applied along [111] $\mathrm{P}$ as shown in Fig. 3.

\section{Discussion}

As described in the thermal expansion measurement and the optical microscope observation, we can say that the specific variant, whose uniaxial magnetocrystalline anisotropy energy is the lowest, is selected to grow by applying the magnetic field.

However, even though we consider the above thing, we can not explain the reason why the variants are rearranged 


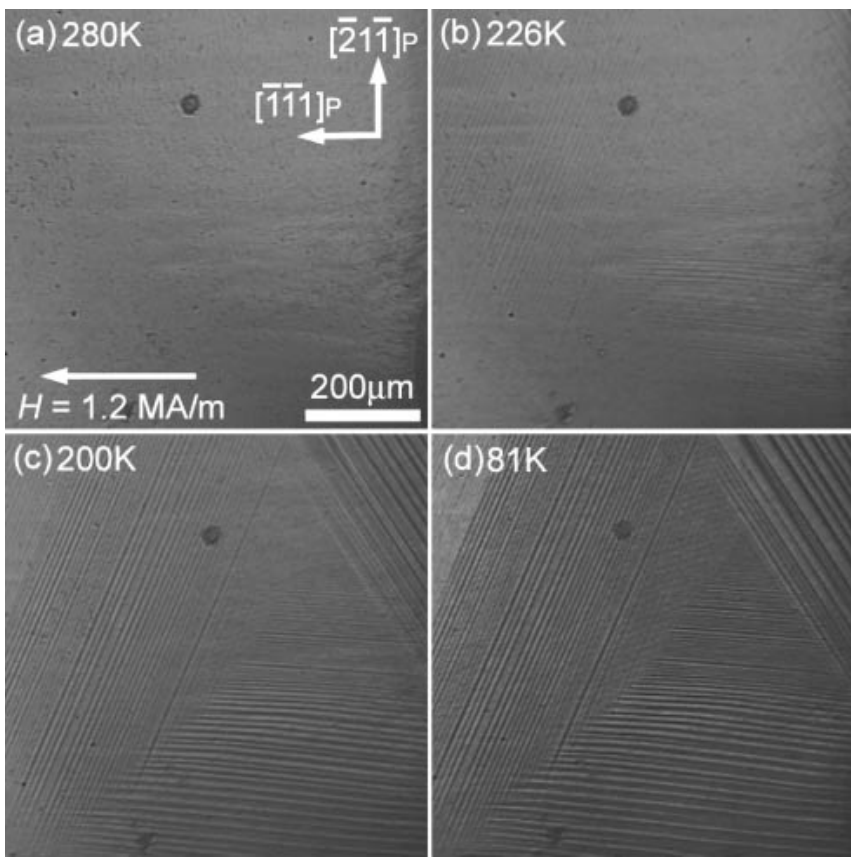

Fig. 6 A series of optical micrographs of $\mathrm{Fe}-31.2 \mathrm{Pd}(\mathrm{at} \%)$ in the cooling process under the magnetic field of $1.2 \mathrm{MA} / \mathrm{m}$ along the $[111]_{\mathrm{P}}$ direction: the parent phase is shown in (a); the martensite phase is shown in (b), (c) and (d).

perfectly into the variants whose $a$ axes are parallel to the magnetic field applied along $[001]_{\mathrm{P}}$ and imperfectly into the variant whose $c$ axis is perpendicular to the magnetic field applied along $[011]_{\mathrm{P}}$. We will discuss it as the following.

In order that rearrangement of variants occurs by a magnetic field, a shear stress should act on the twinning plane. Considering the fact that the energy dissipated due to the rearrangement of variants by a magnetic field is almost the same as that by the external stress, ${ }^{13)}$ we can evaluate a kind of shear stress by the magnetic field. For simplicity, we consider a case in which rearrangement of variants occurs from a single variant to another single variant, although it is a rough approximation. We make such an approximation because the evaluation of the energy of the multi-variant state in the present alloy is complex. The shear stress $\tau_{\text {mag }}$ generated by applying a magnetic field will be expressed as $\Delta U_{\text {mag }} / S$ where $\Delta U_{\text {mag }}$ is the difference of magnetic energy before and after rearrangement of variants and $S$ is the amount of shear. The maximum value of $\Delta U_{\text {mag }}$ is $\left|K_{\mathrm{u}}\right|$ when the magnetic field is applied along $[001]_{\mathrm{P}}$ and $\left|K_{\mathrm{u}}\right| / 2$ when the magnetic field is applied along $[011]_{\mathrm{P}}$. The value of $\left|K_{\mathrm{u}}\right|$ is $350 \mathrm{~kJ} / \mathrm{m}^{3}$ and $S$ is 0.124 at $77 \mathrm{~K} .^{13)}$ Thus the maximum value of $\tau_{\mathrm{mag}}$ is $\left|K_{\mathrm{u}}\right| / S=2.8 \mathrm{MPa}$ when a magnetic field is applied along $[001]_{\mathrm{P}}$, and $\left|K_{\mathrm{u}}\right| / 2 S=1.4 \mathrm{MPa}$ when a magnetic field is applied along $[011]_{\mathrm{P}}$. According to our data of the tensile tests along $[001]_{\mathrm{P}}{ }^{13)}$ and $[011]_{\mathrm{P}},{ }^{14)}$ the shear stress required for rearrangement of variants $\tau_{\text {req }}$ is between 0.5 and $2.5 \mathrm{MPa}$. Thus the maximum value of $\tau_{\text {mag }}$ is always larger than $\tau_{\text {req }}$ when a magnetic field is applied along $[001]_{P}$, but is not always when a magnetic field is applied along $[011]_{\mathrm{P}}$. When a magnetic field is applied along $[111]_{\mathrm{P}}$, $\tau_{\text {mag }}$ is always zero. Therefore the rearrangement of variants is completed when a magnetic field applied along $[001]_{\mathrm{P}}$, it is not completed when $[011]_{\mathrm{P}}$, and it does not occur when
$[111]_{\mathrm{P}}$.

Accordingly, influence of magnetic field direction on rearrangement of variants is explained by using the criterion that a kind of shear stress generated by a magnetic field acting on the twinning plane is larger than the stress required for rearrangement of variants, which is consistent with the criterion Ullakko et al. proposed. ${ }^{4)}$

\section{Conclusion}

The rearrangement of martensite variants in $\mathrm{Fe}-31.2 \mathrm{Pd}$ significantly depends on the direction of magnetic field. When a magnetic field is applied along $[001]_{\mathrm{P}}$, the variants are rearranged almost perfectly into the variants whose $a$ axes are parallel to the magnetic field. When a magnetic field is applied along $[011]_{\mathrm{P}}$, they are rearranged imperfectly into the variant whose $c$ axis is perpendicular to the magnetic field. When a magnetic field is applied along $[111]_{\mathrm{P}}$, they are hardly rearranged. This behavior is explained by the following criterion: rearrangement of variants proceeds when a kind of shear stress generated by applying magnetic field acting on the twinning plane is larger than the stress required for rearrangement of variants.

\section{Acknowledgements}

A part of this work is supported by the Ministry of Education, Culture, Sports, Science and Technology of Japan (MEXT), through MEXT Special Coordination Funds for Promoting Science and Technology (Nanospintronics Design and Realization, NDR; Strategic Research Base's Handai Frontier Research Center). This work was also supported by "Priority Assistance of the Formation of Worldwide Renowned Centers of Research-The 21st Century COE Program (Project: Center of Excellence for Advanced Structural and Functional Materials Design)" from MEXT.

\section{REFERENCES}

1) K. Ullakko, J. K. Huang, C. Kantner, R. C. O'Handley and V. V. Kokorin: Appl. Phys. Lett. 69 (1996) 1966-1968.

2) T. Kakeshita, T. Takeuchi, T. Fukuda, M. Tsujiguchi, T. Saburi, R. Oshima and S. Muto: Appl. Phys. Lett. 77 (2000) 1502-1504.

3) T. Sakamoto, T. Fukuda, T. Kakeshita, T. Takeuchi and K. Kishio: J. Appl. Phys. 93 (2003) 8647-8649.

4) K. Ullakko, J. K. Huang, V. V. Kokorin and R. C. O'Handley: Scr. Mater. 36 (1997) 1133-1138.

5) R. D. James and M. Wuttig: Philos. Mag. A 77 (1998) 1273-1299.

6) R. C. O'Handley: J. Appl. Phys. 83 (1998) 3263-3270.

7) V. A. L'lov, E. V. Gomonaj and V. A. Chernenko: J. Phys.: Condens. Matter. 10 (1998) 4587-4596.

8) R. D. James, R. Tickle and M. Wuttig: Mater. Sci. Eng. A273-275 (1999) 320-325.

9) A. A. Likhachev and K. Ullakko: Phys. Lett. A 275 (2000) 142-151.

10) P. Mülner, V. A. Chernenko, M. Wollgarten and G. Kostorz: J. Appl. Phys. 92 (2002) 6708-6713.

11) J. Koeda, Y. Nakamura. T. Fukuda. T. Kakeshita. T. Takeuchi and K. Kohji: Trans. Mater. Res. Soc. Japan 26[1] (2001) 215-217.

12) M. Sugiyama, R. Oshima and F. E. Fujita: Trans. JIM 25 (1984) 585592.

13) T. Fukuda, T. Sakamoto, T. Tomoyuki, T. Tetusuya and K. Kishio: Mater. Trans., to be published.

14) T. Sakamoto, T. Fukuda, T. Tomoyuki and K. Kishio: to be published. 